BULLETIN OF THE

AMERICAN MATHEMATICAL SOCIETY

Volume 78, Number 4, July 1972

\title{
FILTERED AND ASSOCIATED GRADED RINGS
}

\author{
BY JANE PURCELL COFFEE ${ }^{1}$
}

Communicated by M. Gerstenhaber, January 3, 1972

1. Introduction. The object of this note is to present a condition which guarantees that a filtered ring $A$ is isomorphic (in the category of filtered rings) to its associated graded ring $\mathrm{gr} A$. The result is that a separated, complete, nonnegatively filtered ring $A$ over a field $k$ of characteristic 0 is isomorphic to $\mathrm{gr} A$ if and only if $\operatorname{dim}_{k} H^{2}(\mathrm{gr} A$, gr $A)=\operatorname{dim}_{k} H^{2}(A, A)$ where the $\operatorname{dim}_{k} H^{2}(\mathrm{gr} A, \operatorname{gr} A)$ is finite. The tool is algebraic deformation theory. Rim has observed that an application of the main theorem yields a condition for a plane algebroid curve over an algebraically closed field of characteristic 0 to be of the form $u^{m}=v^{n}$-a result obtained by Zariski [5] by a different approach.

2. Since $A$ is a deformation of $\operatorname{gr} A$ (Gerstenhaber [1]), there exists a one-parameter family of deformations $A_{t}=\mathrm{gr} A[[t]]$ with multiplication defined by $f_{t}(a, b)=a b+t F_{1}(a, b)+t^{2} F_{2}(a, b)+\cdots$. It is known that the deformation from gr $A$ to $A$ given by $A_{t}$ is a "pop deformation", i.e., for $t \neq 0, A_{t}$ is isomorphic as a filtered ring to $A[[t]]$ (Gerstenhaber [2]).

Let $\delta_{t}$ denote the Hochschild coboundary operator of the algebra $A_{t}$, i.e., computed relative to the multiplication $f_{t}$. For example, for $\varphi \in C^{1}\left(A_{t}, A_{t}\right)$, the group of 1-cochains of $A_{t}$, one has

$$
\delta_{t} \varphi(a, b)=f_{t}(a, \varphi b)-\varphi\left(f_{t}(a, b)\right)+f_{t}(\varphi a, b) .
$$

If there exists $\eta_{t} \in C^{1}\left(A_{t}, A_{t}\right)$ such that $z_{t}=\delta_{t} \eta_{t}$, then $z_{t} \in B^{2}\left(A_{t}, A_{t}\right)$. $z_{0} \in Z^{2}(\operatorname{gr} A, \operatorname{gr} A)$ is extendible if there exists $z_{t} \in Z^{2}\left(A_{t}, A_{t}\right)$ such that

$$
z_{t}=z_{0}+t z_{1}+t^{2} z_{2}+t^{3} z_{3}+\cdots
$$

Note that every $b_{0} \in B^{2}(\operatorname{gr} A, \operatorname{gr} A)$ is extendible since $b_{0}=\delta \eta_{0}$ implies that $b_{t}=\delta_{t} \eta_{0}=b_{0}+t b_{1}+t^{2} b_{2}+\cdots$ is an extension of $b_{0}$ where $\eta_{0}$ is extended linearly over $k((t))$. An extendible class of $H^{2}(\operatorname{gr} A, \operatorname{gr} A)$ is a $\left[z_{0}\right]$ for which there is a representative $z_{0}$ which is extendible. $z_{0} \in Z^{2}(\operatorname{gr} A, \operatorname{gr} A)$ is a jump cocycle if there exists an extension $z_{t}$ of $z_{0}$ such that $z_{t} \in B^{2}\left(A_{t}, A_{t}\right)$. Each $b_{0}=\delta \eta_{0} \in B^{2}(\operatorname{gr} A$, gr $A)$ is a jump cocycle since $b_{t}=\delta_{t} \eta_{0}$ is an extension of $b_{0}$ and $b_{t} \in B^{2}\left(A_{t}, A_{t}\right)$. A jump class of $H^{2}(\operatorname{gr} A, \operatorname{gr} A)$ is a $\left[z_{0}\right]$ for which there exists a representative $z_{0}$ which is a jump cocycle.

\footnotetext{
AMS 1970 subject classifications. Primary 16A58; Secondary 18H15.

Key words and phrases. Filtered rings, associated graded rings, algebraic deformations, extendible cocycle, jump cocycle.

1 The results announced here are contained in the author's Ph.D. thesis. written under the guidance of Murray Gerstenhaber at the University of Pennsylvania.
} 
The following theorem is the algebraic analogue of results obtained by Griffiths [3] for normed complexes and for fibered complex-analytic varieties. We assume the vector space dimension, $\operatorname{dim}_{k} H^{2}(\operatorname{gr} A, \operatorname{gr} A)$, is finite.

THEOREM 1.

$$
\begin{aligned}
\operatorname{dim}_{k(t))} H^{2}\left(A_{t}, A_{t}\right) & =\operatorname{dim}_{k} \frac{\text { Extendible classes of } H^{2}(\mathrm{gr} A, \mathrm{gr} A)}{\text { Jump classes of } H^{2}(\operatorname{gr} A, \operatorname{gr} A)} \\
& =\operatorname{dim}_{k} E / J .
\end{aligned}
$$

Proof. To prove that $\operatorname{dim}_{k((t))} H^{2}\left(A_{t}, A_{t}\right) \leqq \operatorname{dim}_{k} E / J$ one shows that a basis $\left[z_{t}^{i}\right], i=1, \ldots, m$, of $H^{2}\left(A_{t}, A_{t}\right)$ over $k((t))$ can be chosen so that $z_{t}^{i}=z_{0}^{i}+t z_{1}^{i}+t^{2} z_{2}^{i}+\cdots,\left[z_{0}^{i}\right]$ are linearly independent over $k$ and $\left\{\left[z_{0}^{i}\right]\right\}$, the coset of $\left[z_{0}^{i}\right]$ in $E / J$, are linearly independent over $k$. The map $\left[z_{t}^{i}\right] \rightarrow\left\{\left[z_{0}^{i}\right]\right\}$ then establishes this inequality. The map: Extendible classes $\rightarrow H^{2}\left(A_{t}, A_{t}\right)$ defined by $\left[z_{0}^{i}\right] \rightarrow\left[z_{t}^{i}\right]$ has kernel equal to the jump classes. An elementary argument shows that this map $E / J \rightarrow H^{2}\left(A_{t}, A_{t}\right)$ preserves linear independence. Thus $\operatorname{dim}_{k} E / J \leqq \operatorname{dim}_{k(t))} H^{2}\left(A_{t}, A_{t}\right)$.

The multiplication of $A_{t}$ has been defined as $f_{t}(a, b)=a b+t F_{1}(a, b)$ $+t^{2} F_{2}(a, b)+\cdots$.

Proposition 1. $F_{1}$ is extendible.

Proof. Define $F_{t}(a, b)=F_{1}(a, b)+2 t F_{2}(a, b)+3 t^{2} F_{3}(a, b)+\cdots . F_{t}$ is an extension of $F_{1}$ since $f_{t}\left(a, f_{t}(b, c)\right)-f_{t}\left(f_{t}(a, b), c\right)=0$ holds and the formal derivative of this is

$$
f_{t}\left(a, F_{t}(b, c)\right)+F_{t}\left(a, f_{t}(b, c)\right)-F_{t}\left(f_{t}(a, b), c\right)-f_{t}\left(F_{t}(a, b), c\right)=0
$$

which is precisely the condition for $F_{t}$ to be a $\delta_{t}$-cocycle.

It is important, as Rim observes, that $F_{t}$, the derivative of the multiplication $f_{t}$, not only is a cocycle of the deformed algebra but is actually intrinsic to the deformed algebra and represents a cohomology class which would not be altered if $f_{t}$ were replaced by an equivalent multiplication $g_{t}$. This is proved by the following observations. If $f_{t}$ and $g_{t}$ are equivalent multiplications, then

$$
f_{t}(a, b)=\psi_{t}^{-1}\left(g_{t}\left(\psi_{t} a, \psi_{t} b\right)\right)
$$

where $\psi_{t}$ is a linear automorphism. The formal derivative of $\psi_{t} f_{t}(a, b)$ $=g_{t}\left(\psi_{t} a, \psi_{t} b\right)$ is

(2) $\psi_{t}^{\prime}\left(f_{t}(a, b)\right)+\psi_{t} F_{t}(a, b)=G_{t}\left(\psi_{t} a, \psi_{t} b\right)+g_{t}\left(\psi_{t}^{\prime} a, \psi_{t} b\right)+g_{t}\left(\psi_{t} a, \psi_{t}^{\prime} b\right)$

where $G_{t}$ is the derivative of $g_{t}$. From (1) and (2) it follows that

$$
\begin{aligned}
& \psi_{t}^{-1} \psi_{t}^{\prime}\left(f_{t}(a, b)\right)+F_{t}(a, b) \\
& \quad=\psi_{t}^{-1} G_{t}\left(\psi_{t} a, \psi_{t} b\right)+f_{t}\left(\psi_{t}^{-1} \psi_{t}^{\prime} a, b\right)+f_{t}\left(a, \psi_{t}^{-1} \psi_{t}^{\prime} b\right) .
\end{aligned}
$$


Therefore $F_{t}(a, b)=\psi_{t}^{-1} G_{t}\left(\psi_{t} a, \psi_{t} b\right)+\delta_{t} \psi_{t}^{-1} \psi_{t}^{\prime}(a, b)$ where $\delta_{t}$ is defined with respect to $f_{t}$ multiplication and the cohomology class in $H^{2}\left(A_{t}, A_{t}\right)$ determined by $F_{t}$ is not altered by a change of basis.

Proposition 2. $F_{1}$ is a jump cocycle.

Proof. Let $\Phi_{t}$ be an algebra isomorphism of $A_{t}$ onto $A_{1}$ where $t \neq 0$. Then $\Phi_{t}(f(a, b))=f_{1}\left(\Phi_{t} a, \Phi_{t} b\right)$ and the derivative of both sides of this expression is

$$
\Phi_{t}^{\prime}\left(f_{t}(a, b)\right)+\Phi_{t}\left(F_{t}(a, b)\right)=f_{1}\left(\Phi_{t}^{\prime} a, \Phi_{t} b\right)+f_{1}\left(\Phi_{t} a, \Phi_{t}^{\prime} b\right)
$$

where $\Phi_{t}^{\prime}$ is the formal derivative of $\Phi_{t}$. Rewriting this expression yields

$$
F_{t}(a, b)=f_{t}\left(\Phi_{t}^{-1} \Phi_{t}^{\prime} a, b\right)-\Phi_{t}^{-1} \Phi_{t}^{\prime}\left(f_{t}(a, b)\right)+f_{t}\left(a, \Phi_{t}^{-1} \Phi_{t}^{\prime} b\right)
$$

and thus $F_{t}=\delta_{t} \Phi_{t}^{-1} \Phi_{t}^{\prime}$.

THEOREM 2. A separated, complete filtered ring $A$ over a field $k$ of characteristic 0 is isomorphic to $\operatorname{gr} A$ if and only if $\operatorname{dim}_{k} H^{2}(\mathrm{gr} A, \mathrm{gr} A)$ $=\operatorname{dim}_{k} H^{2}(A, A)$ where the vector space $\operatorname{dim}_{k} H^{2}(\operatorname{gr} A, \operatorname{gr} A)$ is finite.

Proof. By $[2], A[[t]]$ is isomorphic to $A_{t}$ for $t \neq 0$. The

$$
\operatorname{dim}_{k(t))} H^{2}(A[[t]], A[[t]])=\operatorname{dim}_{k} H^{2}(A, A) .
$$

It is therefore sufficient to prove that, for $t \neq 0, A_{t}$ is isomorphic to gr $A[[t]]$ with multiplication $f_{0}$ if $\operatorname{dim}_{k} H^{2}(\operatorname{gr} A$, gr $A)=\operatorname{dim}_{k(t))} H^{2}\left(A_{t}, A_{t}\right)$. By Theorem 2,

$$
\begin{aligned}
\operatorname{dim}_{k((t))} H^{2}\left(A_{t}, A_{t}\right) & =\operatorname{dim}_{k} \frac{\text { Extendible classes of } H^{2}(\operatorname{gr} A, \operatorname{gr} A)}{\text { Jump classes of } H^{2}(\operatorname{gr} A, \operatorname{gr} A)} \\
& \leqq \operatorname{dim}_{k} H^{2}(\operatorname{gr} A, \operatorname{gr} A) .
\end{aligned}
$$

Therefore the $\operatorname{dim}_{k(t))} H^{2}\left(A_{t}, A_{t}\right)=\operatorname{dim}_{k} H^{2}(\operatorname{gr} A, \operatorname{gr} A)$ implies that the jump classes of $H^{2}(\mathrm{gr} A, \mathrm{gr} A)=$ coboundaries $\}$. But $F_{1}$ is a jump cocycle. Thus $F_{1}=\delta \rho_{1}$ and $P_{t}(a)=a-t \rho_{1}(a)$ is an isomorphism of $A_{t}$ to $\mathrm{gr} A[[t]]$ with multiplication $a b+t^{2} F_{2}(a, b)+t^{3} F_{3}(a, b)+\cdots$. Provided $k$ has characteristic 0 the above argument can be repeated for $F_{2}$ and, in general, for $F_{n}$ to show that $F_{n}$ is a jump cocycle with the derivative of the appropriate multiplication taken as the extension of $F_{n}$. By the assumption on dimension, $F_{n}=\delta \rho_{n}$. Therefore $A_{t}$ is isomorphic to gr $A[[t]]$ with multiplication $f_{0}$.

3. Let $k$ be an algebraically closed field of characteristic 0 and let $f(x, y)$ be an irreducible power series with coefficients in $k$. Let $C$ be the plane curve defined by $f=0, A$ be the local ring of $C$ and $m_{A}$ be the maximal ideal of $A$. 
The Weierstrass Preparation Theorem and Puiseux's Theorem together imply that $A \subset k[[t]]$. Thus we can define a filtration on $A$ so that $F_{0} A=A \supset F_{1} A=t \cap A \supset F_{2} A=t^{2} \cap A \supset \cdots$ and form the associated graded ring $\mathrm{gr} A$. We may assume $\operatorname{gr}_{1} A=F_{1} A / F_{2} A=0$ since otherwise $t \in A$ implies that $A=k[[t]]$ and the curve $C$ would be nonsingular.

gr $A=k\left[\left[t^{v_{1}}, t^{v_{2}}, \ldots t^{v_{r}}\right]\right]$ with $v_{1}<v_{2}<\cdots<v_{r}$ by definition of the filtration on $A$. Since $A$ is the local ring of a plane algebroid curve, gr $A$ is generated by at most two elements.

The main result of $\S 1$ states that $A$ is isomorphic to $\mathrm{gr} A$ if and only if $\operatorname{dim}_{k} H^{2}(\operatorname{gr} A, \operatorname{gr} A)=\operatorname{dim}_{k} H^{2}(A, A)$. Thus a suitable basis $\{u, v\}$ of $m_{A}$ can be chosen so that the curve $C$ is of the form $u^{m}=v^{n}$ provided that $\operatorname{dim}_{k} H^{2}(\operatorname{gr} A, \operatorname{gr} A)=\operatorname{dim}_{k} H^{2}(A, A)$. Rim has observed that these results give an alternate form to a result of Zariski [5] which states that $l(T)=L$ if and only if for a suitable basis $\{x, y\}$ of $m_{A}$ the equation of the curve $C$ is of the form $y^{n}=x^{m}$ where $(n, m)=1$ by the irreducibility of the curve $C, l(T)$ is the length of the $A$-module $T$ ( $T$ is the torsion submodule of the module of Kähler differentials of $A$ ) and $L$ is the length of the conductor of $A$ in the integral closure $\bar{A}$ of $A$.

\section{REFERENCES}

1. M. Gerstenhaber, On the deformation of rings and algebras, Ann. of Math. (2) 79 (1964), 59-103. MR 30 \#2034.

2. , On the deformation of rings and algebras, II, Ann. of Math. (2) 84 (1966), 1-19. MR 34 \# 7608.

3. P. A. Griffiths, The extension problem for compact submanifolds of complex manifolds. I. The case of a trivial normal bundle, Proc. Conference Complex Analysis (Minneapolis, 1964), Springer, Berlin, 1965, pp. 113-142. MR 32 \#8362.

4. R. M. Walker, Algebraic curves, Princeton Math. Series, vol. 13, Princeton Univ. Press, Princeton, N.J., 1950. MR 11, 387.

5. O. Zariski, Characterization of plane algebroid curves whose module of differentials has maximum torsion, Proc. Nat. Acad. Sci. U.S.A. 56 (1966), 781-786. MR 34 \# 2576.

6. O. Zariski and P. Samuel, Commutative algebra. Vol. II, The University Series in Higher Math., Van Nostrand, Princeton, N.J., 1960. MR 22 \# 11006.

Department of Mathematics, Richmond College of the City University of New YORK, STATEN ISLAND, NEW YORK 10301 